\title{
A novel COL1A1 mutation in infantile cortical hyperostosis (Caffey disease) expands the spectrum of collagen-related disorders
}

\author{
Robert C. Gensure, ${ }^{1}$ Outi Mäkitie, ${ }^{2,3}$ Catherine Barclay, ${ }^{2}$ Catherine Chan, ${ }^{2}$ \\ Steven R. DePalma, ${ }^{4}$ Murat Bastepe, ${ }^{1}$ Hilal Abuzahra, ${ }^{1}$ Richard Couper, ${ }^{5}$ Stefan Mundlos, ${ }^{6}$ \\ David Sillence, ${ }^{7}$ Leena Ala Kokko, ${ }^{8}$ Jonathan G. Seidman, ${ }^{4}$ William G. Cole, ${ }^{2}$ and Harald Jüppner ${ }^{1}$

\begin{abstract}
${ }^{1}$ Endocrine and Pediatric Endocrine Units, Departments of Medicine and Pediatrics, Massachusetts General Hospital and Harvard Medical School, Boston, Massachusetts, USA. ${ }^{2}$ Division of Orthopaedics, The Hospital for Sick Children, Toronto, Ontario, Canada. ${ }^{3}$ Department of Pediatric Endocrinology, Helsinki University Hospital, Helsinki, Finland. ${ }^{2}$ Department of Genetics, Harvard Medical School and Howard Hughes Medical Institute, Boston, Massachusetts, USA. 'University of Adelaide, Department of Paediatrics, Women's \& Children's Hospital, North Adelaide, South Australia, Australia. ${ }^{6}$ Max-Planck Institute for Molecular Genetics and Institute for Medical Genetics, Berlin, Germany. 'Departments of Paediatrics and Child Health, The Children's Hospital at Westmead Clinical School, Westmead, New South Wales, Australia. ${ }^{8}$ Collagen Research Unit, Biocenter and Department of Medical Biochemistry and Molecular Biology, University of Oulu, Oulu, Finland.
\end{abstract}

\begin{abstract}
Infantile cortical hyperostosis (Caffey disease) is characterized by spontaneous episodes of subperiosteal new bone formation along 1 or more bones commencing within the first 5 months of life. A genome-wide screen for genetic linkage in a large family with an autosomal dominant form of Caffey disease (ADC) revealed a locus on chromosome 17q21 (LOD score, 6.78). Affected individuals and obligate carriers were heterozygous for a missense mutation $(3040 \mathrm{C} \rightarrow \mathrm{T}$ ) in exon 41 of the gene encoding the $\alpha 1(\mathrm{I})$ chain of type I collagen (COL1A1), altering residue $836(\mathrm{R836C})$ in the triple-helical domain of this chain. The same mutation was identified in affected members of 2 unrelated, smaller families with ADC, but not in 2 prenatal cases and not in more than 300 chromosomes from healthy individuals. Fibroblast cultures from an affected individual produced abnormal disulfide-bonded dimeric $\alpha 1$ (I) chains. Dermal collagen fibrils of the same individual were larger, more variable in shape and size, and less densely packed than those in control samples. Individuals bearing the mutation, whether they had experienced an episode of cortical hyperostosis or not, had joint hyperlaxity, hyperextensible skin, and inguinal hernias resembling symptoms of a mild form of Ehlers-Danlos syndrome type III. These findings extend the spectrum of COL1A1-related diseases to include a hyperostotic disorder.
\end{abstract}

\section{Introduction}

Infantile cortical hyperostosis (Caffey disease; OMIM 114000) is a genetic disorder characterized by an infantile episode of massive subperiosteal new bone formation that typically involves the diaphyses of the long bones, mandible, and clavicles (1). In addition to these changes, which can appear quite prominently on x-ray, the involved bones may also appear inflamed, with painful swelling and systemic fever often accompanying the illness. Consistent with the observed increased bone formation, laboratory findings include an elevated level of alkaline phosphatase. Furthermore, there can be an elevation in white blood cell count and erythrocyte sedimentation rate, which indicate an inflammatory response. The bone changes usually begin before 5 months of age and resolve before 2 years of age $(1,2)$. Recurrent episodes of cortical hyperostosis are uncommon, and there are few reports concerning adverse sequelae of the hyperostotic lesions in affected individuals $(3,4)$. The inflammatory nature of the symptoms has led to treatment trials with antiinflammatory agents, and resolution of symptoms has been reported with the use of indomethacin (5) and with glucocorticoid therapy (6).

Nonstandard abbreviations used: ADC, autosomal dominant form of Caffey disease; COL1A1, gene encoding the $\alpha 1$ (I) chain of type I collagen; CB6, cyanogen bromide peptide 6; PGE1, prostaglandin E1.

Conflict of interest: The authors have declared that no conflict of interest exists.

Citation for this article: J. Clin. Invest. 115:1250-1257 (2005).

doi:10.1172/JCI200522760.
Information on several unrelated pedigrees indicates that Caffey disease can be inherited in an autosomal dominant manner (7-9). Review of these pedigrees showed evidence of incomplete penetrance, as there were obligate carriers who lacked episodes of cortical hyperostosis (7-9). However, the signs and symptoms of Caffey disease can be subtle and are typically only manifested at a preverbal age, which thus makes it plausible that the diagnosis could have been missed in some of these apparently unaffected carriers. A more severe and often lethal prenatal form of Caffey disease appears to be inherited as an autosomal recessive disorder (10).

In addition to the inherited forms of Caffey disease, sporadic cases of infantile cortical hyperostosis have also been described (reviewed in ref. 11). At least some of these nonfamilial cases can be attributed to administration of prostaglandin E1 (PGE1) and PGE2 for treatment of ductal-dependent cardiac lesions (12-14). In fact, the incidence of this complication of prostaglandin therapy is high, with radiographic changes occurring in $62 \%$ of all infants receiving PGE1 infusion for more than 60 days (14). Other conditions that can cause cortical bone lesions in children less than 2 years of age, and may thus mimic infantile cortical hyperostosis, include trauma (15), hypervitaminosis A (16), hyperphosphatemia (17), and infection (18). Sporadic cases of infantile cortical hyperostosis not associated with PGE1 or PGE2 infusion appear to be on the decline, but the reason(s) for the diminished frequency of these bone abnormalities remain(s) uncertain (8). 

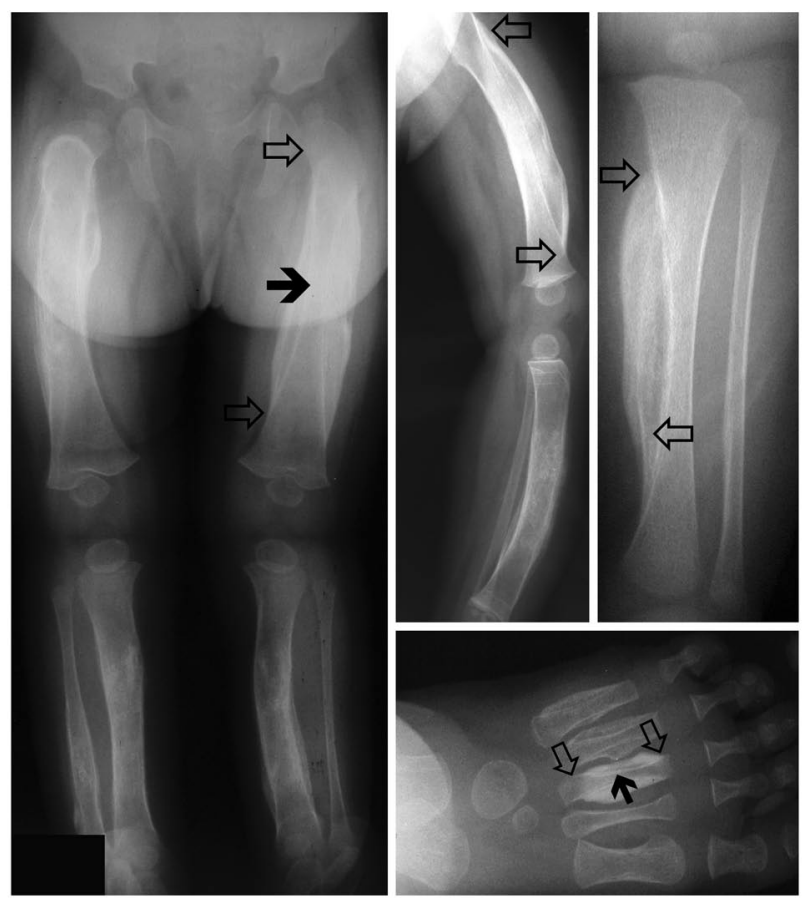

The gene or genes responsible for the prenatal and postnatal inherited forms of Caffey disease have not been reported. We therefore undertook genetic linkage studies using genomic DNA from a large kindred with the autosomal dominant form of the condition (autosomal dominant Caffey disease $[\mathrm{ADC}])(7,8)$. Affected individuals and obligate carriers in this kindred, as well as affected individuals in 2 other kindreds, were shown to be heterozygous for the same novel missense mutation affecting the gene that encodes the $\alpha 1(\mathrm{I})$ chain of type I collagen (COL1A1).

\section{Results}

Genetic linkage analysis. Three unrelated families with at least 2 members affected by $\operatorname{ADC}(7,8,19)$ (Figure 1$), 1$ sporadic case, and 2 prenatal cases were available for analysis $(20,21)$. DNA from family 1 was used for a genome-wide scan, which revealed linkage to chromosome 17q21 (Figure 2A). Fine mapping reduced the size of the linked region to a $2.3-\mathrm{Mb}$ interval delimited by markers D17S1868 and D17S1877. The maximal LOD score obtained within this region by 2 -point analysis was 6.78 for marker $D 17 S 1795(\theta=0)$, which is only slightly lower than the maximal theoretical LOD score of 7.12. Nucleotide sequence analysis of 13 candidate genes within the linked region (GNGT2, NESH, PHB, LOC81558, ITGA3, LOC84687, XT2, PRO1855, CHAD, EPN3, FLJ21347, OA48-18, and TOB1) did not reveal any heterozygous mutations that segregated with ADC. However, affected individuals and obligate carriers were heterozygous for a missense mutation in exon 41 of COL1A1 (also referred to as exon 42, corresponding to the exon structure of COL1A2, which contains 1 additional exon; ref. 22) (Figure 3). This $\mathrm{C} \rightarrow \mathrm{T}$ transition at nucleotide position 3040 of the cDNA (relative to the translation initiation codon), which alters codon 1014 of prepro- $\alpha 1$ (I) mRNA, was predicted to produce an R836C amino acid substitution within the triple-helical domain of the $\alpha 1$ (I) chain of collagen. The nucleotides were numbered relative to the

\section{Figure 1}

Radiographic features of infantile cortical hyperostosis. Radiographs of 3 affected individuals of 2 unrelated kindreds showing subperiosteal thickening of the femur, tibia, and fibula (left and upper middle panels: tibia and fibula, respectively, of twin II-1 of family 3; upper right panel: fibula of patient II-1 of family 2), and metacarpals of the left foot (lower right panel: twin II-2 of family 3). Note that the periosteum, which is normally anchored at the growth plates where it is continuous with the perichondrium, was frequently elevated circumferentially from the proximal to the distal growth plates (open arrows). The bone marrow cavities were also narrowed (filled arrows).

ATG start site of prepro- $\alpha 1$ (I) cDNA, and amino acid residues were numbered relative to the first glycine residue of the main triple-helical domain of the $\alpha 1$ (I) protein chain (22).

To confirm the mutation identified by sequence analysis, a 495-bp fragment comprising exon 41 was PCR amplified and digested with HpyCH4IV (ACGT). The PCR product derived from the mutant allele lacked 1 recognition site for HpyCH4IV, thus generating an additional DNA fragment of $319 \mathrm{bp}$ (Figure 2B). The same COL1A1 mutation was also found in the 3 affected members but not in the unaffected father of family 2 (Figure 2C). Furthermore, the mutation was found in the clinically affected identical twins but not in the clinically unaffected parents and brother of family 3 (Figure 2D). The affected twins and the unaffected sibling had inherited identical parental alleles in this region. Consequently, the clinically affected twins were likely to have acquired a de novo COL1A1 mutation.

Haplotype analysis of the affected members of families 1-3 did not reveal any shared alleles, which supported the historical evidence that the 3 families that were heterozygous for the R836C substitution were not related to each other. The $3040 \mathrm{C} \rightarrow \mathrm{T}$ transition was not detected in genomic DNA from more than 150 healthy controls (more than 300 chromosomes), in SNP databases (dbSNP, http://www.ncbi.nlm.nih.gov/SNP; JSNP, http://snp. ims.u-tokyo.ac.jp; Celera Discovery System, http://publication. celera.com/humanpub/index.jsp; and Applied Biosystems SNPbrowser Software, http://marketing.appliedbiosystems. $\mathrm{com} / \mathrm{mk} / \mathrm{get} / \mathrm{snpb}$ landing), or in the Mutations in COL1A1 database (http://www.le.ac.uk/genetics/collagen/col1a1.html) (22). The latter findings suggest that the $3040 \mathrm{C} \rightarrow \mathrm{T}$ transition in exon 41 of COL1A1 and the resulting R836C amino acid substitution within the triple-helical domain of the $\alpha 1$ (I) chain can be a cause of ADC.

A possible sporadic case of Caffey disease with hyperostosis of a rib did not have any demonstrable mutations in COL1A1 or in the COL1A2 gene, which encodes the $\alpha 2$ (I) chain of type I collagen. Mutations of COL1A1 or COL1A2 were also not detected in 2 cases of prenatal Caffey disease $(20,21)$.

Dermal collagen morphology and collagen biosynthesis. To further investigate whether the Col1A1 mutation results in changes in type I collagen, we obtained skin biopsies from an affected adult female in family 1 (IV-2). This individual had an episode of infantile cortical hyperostosis and from early childhood had clinical signs of hyperextensible skin and generalized joint instability, as well as a history of several fractures. As an adult, she had the same clinical signs, as well as more severe voluntary subluxation of the shoulders. Electron microscopy of her dermis showed normal fibroblast morphology (data not shown). The collagen fibrils were more variable in shape and size and were less densely 


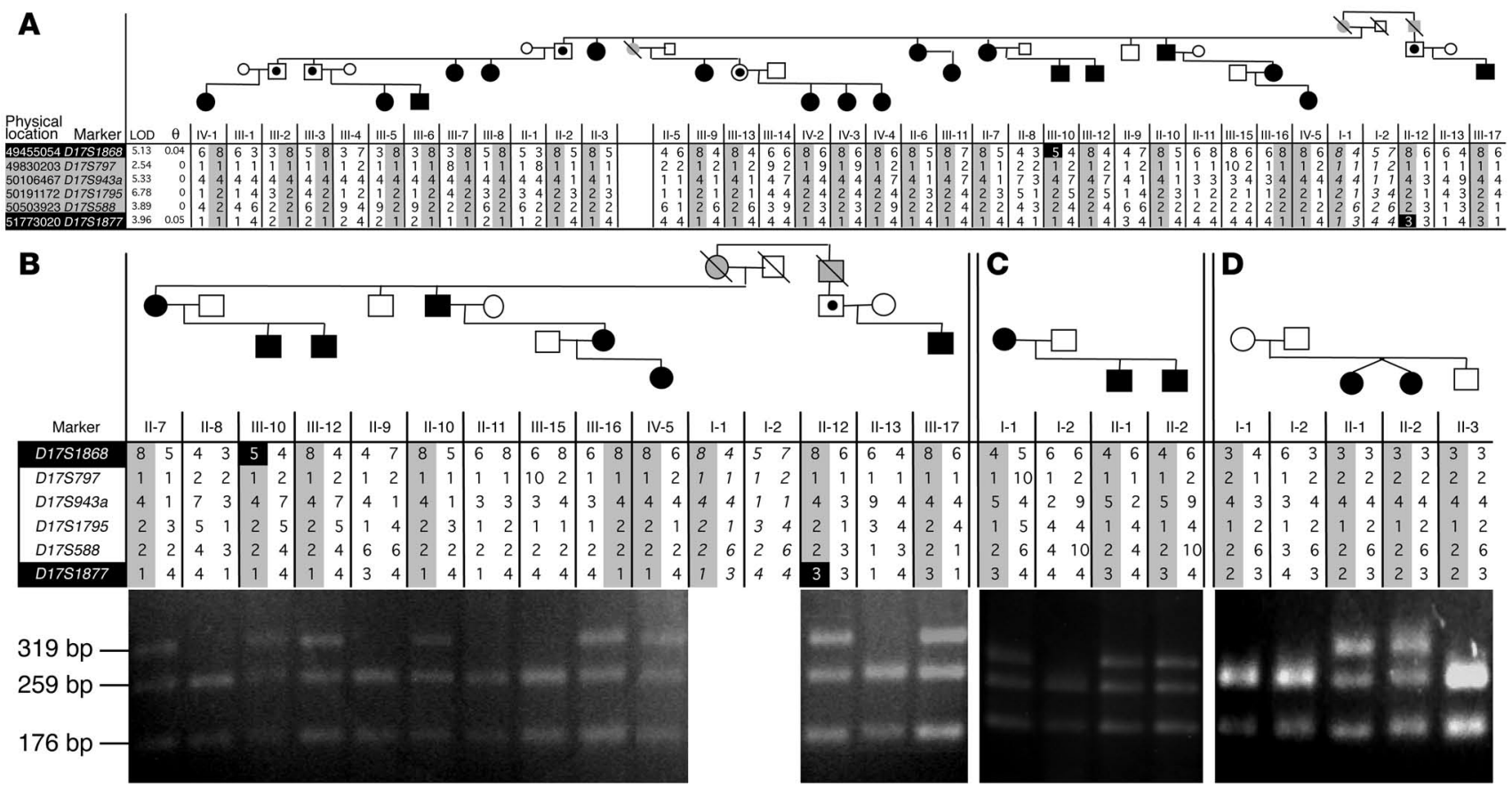

\section{Figure 2}

Haplotype analyses of families with infantile cortical hyperostosis. (A) Family 1: Fine mapping of the locus for Caffey disease on chromosome 17q21. Standard techniques were used to establish the genetic locus of the disease in a large Canadian family with ADC (7, 8). Black symbols, affected individuals; white symbols with black dot in the center, obligate carriers; white symbols, unaffected individuals; gray symbols, deceased individuals. The unaffected individual II-9, who has unaffected children and grandchildren (data not shown), was only included to deduce the haplotypes of I-1 and I-2 (indicated by italics); for LOD score calculations, his phenotype was entered as unknown. Marker D17S1795 provided a LOD score of 6.78 (maximal theoretical LOD score, 7.12). Markers D17S1868 and D17S1877 (indicated in white on a black background) define the centromeric and telomeric boundary, respectively. The disease-associated haplotype is shown by black numbers on gray; markers consistent with a recombination are shown by white numbers on black. Uninformative data and haplotypes not associated with the disease are shown by black numbers on white. A C $\rightarrow$ T mutation at nucleotide 3040 of COL1A1 (see Figure 3) was identified by direct nucleotide sequence analysis only in affected members and obligate carriers. (B) Haplotypes of portions of the family shown in A and PCR-based confirmation of the identified mutation (see Methods). (C) Haplotypes and PCR analysis for family 2, comprising 2 affected brothers, their affected mother, and their healthy father. (D) Haplotypes and PCR analysis for family 3, comprising identical twin sisters affected by Caffey disease and their healthy parents and brother (19).

packed (Figure 4A) than fibrils in age- and sex-matched control dermal samples (Figure 4B). The fibrils were also significantly larger and were more variable in size in the affected individual (Caffey disease, $108 \pm 15 \mathrm{~nm}$; control, $91 \pm 8 \mathrm{~nm}$; $n=1,000$; $P<0.001)$. Granular material was visible in the matrix surrounding many of the collagen fibrils (Figure 4A).

One-dimensional gel electrophoresis showed that cultured dermal fibroblasts from the proband of family 1 produced a mixture of apparently normal type I, III, and V collagens as well as an abnormal disulfide-linked dimer (Figure 5A). As the dimer was likely to include $2 \alpha 1$ (I) chains bearing the R836C substitution, it was designated $\beta 11^{\prime}$. Two-dimensional gel electrophoresis showed that the abnormal $\beta 11^{\prime}$ dimers dissociated after reduction of disulfide bonds into $\alpha 1$ (I) chains that were designated as $\alpha 1(\mathrm{I})^{\prime}$ chains (Figure $5 \mathrm{~B}$ ). Although $\beta$-aminopropionitrile, an inhibitor of the formation of lysine-derived collagen crosslinkages, was added to the cultures, a small amount of dimeric collagen chains ( $\beta 11$ and $\beta 12$ ) was formed. These lysine-derived crosslinked dimers do not dissociate into $\alpha$ chains after reduction of disulfide bonds with dithiothreitol.

Genotype/phenotype correlations. Of the 24 members of family 1 in our study with the R836C mutation, only 19 members had experienced an episode of cortical hyperostosis, while 5 were obligate carriers. Thus, $79 \%$ of the individuals who were heterozygous for the COL1A1 mutation had an episode of cortical hyperostosis, and $21 \%$ of them did not. Detailed interviews of family members did not reveal any precipitating events other than the consistent report that the onset of cortical hyperostosis usually occurred before 5 months of age. None of the children had been given prostaglandins, which can induce subperiosteal new bone formation in neonates (23). The clinical course of the hyperostotic episode was often protracted but resolved after about 2 years. By mid- to late childhood, the hyperostotic long bones in the affected children of families 2 and 3 had remodeled extensively. However, in each affected bone, the central portion of the diaphysis, corresponding to the complete diaphysis of infancy, showed mild external and internal thickening of the cortex. In contrast, the diaphyseal and metaphyseal bone above and below the central region, which would have formed after the episode of hyperostosis, was radiographically normal (data not shown). The epiphyses and growth plates were also radiographically normal.

In family 1, individuals with the R836C substitution (affected individuals and obligate carriers) had varying degrees of joint hyperlaxity that was first observed in early childhood (24). The hyperlaxity was apparent in large and small joints and allowed some individuals bearing the Caffey allele to voluntarily sublux 


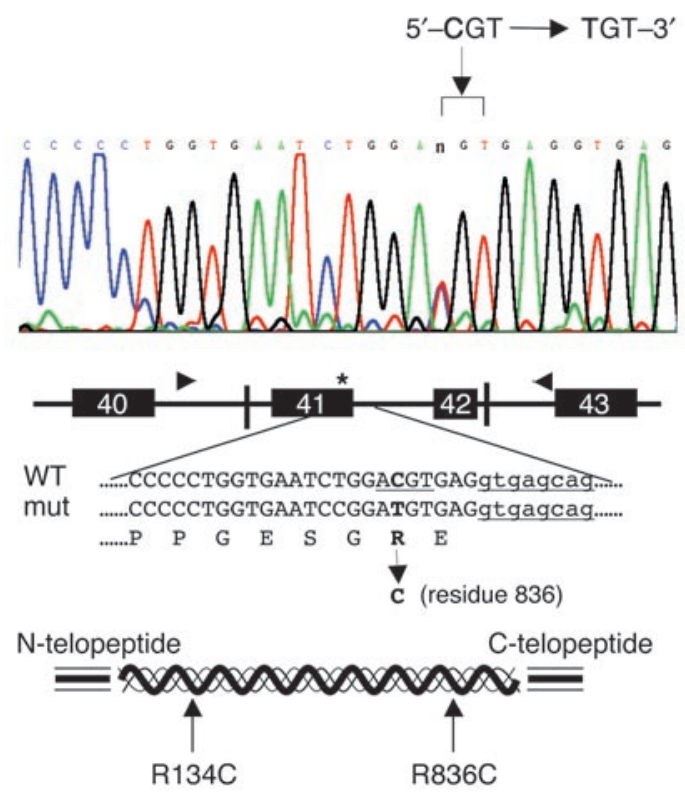

their shoulders and finger joints (Figure 6). Instability of the joints, particularly the shoulder joints, worsened with age. These individuals had abnormally soft and hyperextensible skin, which was otherwise normal in appearance. They did not bruise easily. Members of family 1 who did not bear the Caffey allele did not have the skin or joint anomalies. The 3 members of family 2 with the R836C mutation (see Figure 2C) also had hyperextensible skin, joint hyperlaxity, and inguinal hernias, while the identical twins in family 3 did not demonstrate such anomalies.

None of the affected individuals or obligate carriers in any of the families had clinical signs of the major type I collagen disorder, osteogenesis imperfecta (25). In particular, they lacked grayblue sclerae, dentinogenesis imperfecta, premature hearing loss, short stature, deformities, and scoliosis $(25,26)$. Skull radiographs from the clinically affected identical twins did not contain wormian bones, which can occur in patients with osteogenesis imperfecta $(25,26)$. Neither of the twins had radiographic evidence of platyspondyly, which is also a common feature of osteogenesis imperfecta. In family 1, 9 of the 18 individuals with Caffey disease, for whom detailed fracture histories were available, had a total of 14 peripheral fractures (1-4 in each). Bone mineral density was assessed in 1 affected adult with Caffey disease from family 1 and the results were within normal limits for the lumbar spine and femoral neck; spinal radiographs in this patient showed no evidence of platyspondyly or compression fractures.

\section{Discussion}

In family 1 , we mapped ADC to an interval on chromosome 17q21. All affected individuals and obligate carriers were heterozygous for a 3040C $\rightarrow$ T transition in a CPG dinucleotide of exon 41 of COL1A1, which encodes the $\alpha 1$ (I) chain of type I collagen. The same $3040 \mathrm{C} \rightarrow \mathrm{T}$ transition was also present in affected members of families 2 and 3 . The recurrent nature of the mutation indicates that the involved CpG dinucleotide is a mutational "hot spot" in COL1A1 (27-29). The mutation is predicted to introduce an R836C substitution into the triple-helical domain of $\alpha 1$ (I) chains of type I collagen (22). Results from collagen biosynthetic studies supported this proposal, as cultured dermal fibroblasts from an affected individual pro-

\section{Figure 3}

Nucleotide sequence analysis of portions of exon 41 of COL1A1 and adjacent intronic regions of an affected individual from family 1 . The individual was heterozygous for the transition, which is indicated by the arrow, and the approximate location of both primers for PCR amplification is indicated by arrowheads within the schematic, partial drawing of COL1A1. Partial nucleotide sequence of wild-type and mutant (mut) exon 41 (capital letters), as well as adjacent intronic sequence (lowercase letters), along with the encoded amino acid sequence, are shown. The $3040 \mathrm{C} \rightarrow T$ transition (indicated by an asterisk), which alters the first nucleotide of the second-to-last codon in exon 41 , is predicted to result in the substitution of an arginine $(R) 836$ to a cysteine $(C)$ residue (R836C). The approximate location of the R836C mutation within the triple-helical region of the collagen fibril is schematically shown [bottom: thin lines, $\alpha 1(\mathrm{I})$ chains; thick line, $\alpha 2(\mathrm{I})$ chain], as is the location of the $\mathrm{R} 134 \mathrm{C}$ mutation previously described in 2 unrelated patients with Ehlers-Danlos syndrome type I (31).

duced an abnormal disulfide-bonded $\alpha 1$ (I) dimer. Ultrastructural studies of the dermis revealed that in vivo, the mutation was associated with an abnormal collagen fibril architecture.

The absence of the nucleotide change in any of the databases of normal DNA sequence variants as well as in normal DNA samples supports our proposal that it is a cause of ADC. While the R836C mutation therefore appears to be required for the development of $\mathrm{ADC}$, it remains uncertain how the identified amino acid change can result in a spatially and temporally limited increase in cortical bone formation. COL1A1 and COL1A2 mutations were not found in a sporadic case of Caffey disease in which, according to radiography, changes were limited to a single rib, which suggests that other genetic defects or environmental factors can lead to localized hyperostosis. Mutations of the type I collagen genes were also not detected in 2 cases of the prenatal form of the disease, which is usually more severe and may be inherited in an autosomal recessive manner (21). Consequently, it is likely that the prenatal form of Caffey disease constitutes a separate disorder (11).

The R836C amino acid substitution changes the $\mathrm{X}$ position of one of the 338 Gly-X-Y repeating amino acid triplets of the triple-helical domain of the $\alpha 1$ (I) chain of type I collagen.
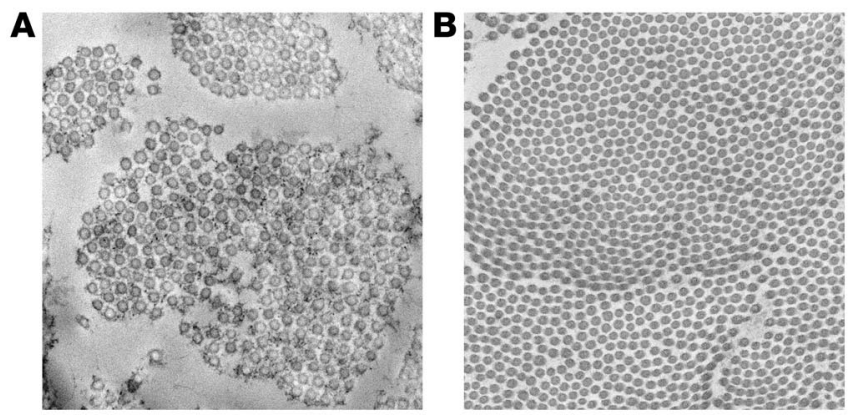

\section{Figure 4}

Dermal ultrastructure from proband IV-2 of family 1. (A) Ultrastructure of the extracellular matrix of the proband's dermis (magnification, $\times 60,000$ ). The collagen fibrils are more variable in shape and size and are less densely packed than in control samples. The fibrils are also larger (Caffey disease, $108 \pm 15 \mathrm{~nm}$; control, $91 \pm 8 \mathrm{~nm} ; n=1,000 ; P<0.0001$ ). Granular material is visible in the matrix surrounding the collagen fibrils. (B) Ultrastructure of control dermis. In contrast to the proband's dermis, the collagen fibrils in the control dermis are round, uniform in size, tightly packed, and are not surrounded by granular material. 
A

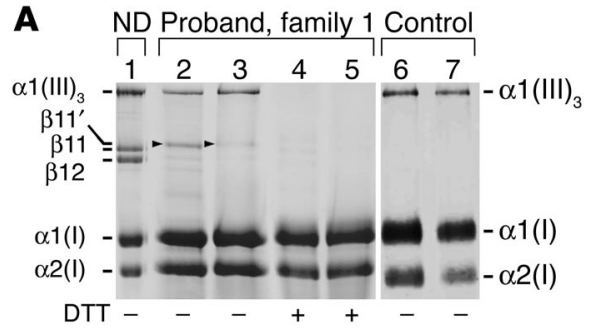

B

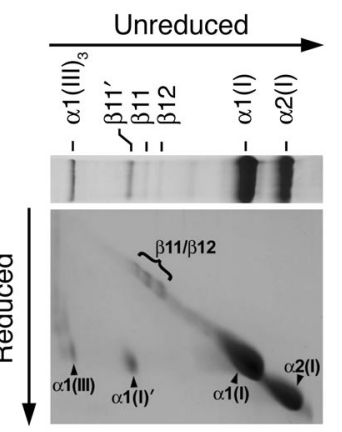

\section{Figure 5}

Collagen biosynthesis by cultured fibroblasts from proband IV-2 of family 1. (A) One-dimensional gel electrophoresis of dermal and fibroblast collagens: lane 1, pepsin-solubilized collagen from normal dermis (ND); lane 2, proband fibroblast cell layer collagens; lane 3, proband medium collagens; lane 4, reduced proband fibroblast cell layer collagens; lane 5, reduced proband medium collagens; lane 6 , control fibroblast cell layer collagens; lane 7 , control medium collagens. All samples contained $\alpha 1(\mathrm{I})$ and $\alpha 2(\mathrm{I})$ monomeric chains of type I collagen. Control dermis (lane 1) contained $\alpha 1(\mathrm{I})$ dimers $(\beta 11)$ and $\alpha 1(\mathrm{I}) / \alpha 2(\mathrm{I})$ dimers $(\beta 12)$ with lysinederived cross-linkages; these cross-linkages were partially blocked with the addition of $\beta$-aminopropionitrile in all fibroblast cultures. The unreduced dermal and fibroblast culture samples contained disulfide-bonded type III collagen trimers $\left[\alpha 1(\mathrm{III})_{3}\right]$. There was an additional protein band in the unreduced proband samples (arrowheads, lanes 2 and 3), designated $\beta 11^{\prime}$, which was more abundant in the cell layer than in the medium. This band migrated slightly slower than the dermal $\beta 11$ dimer. The abnormal band disappeared, along with the type III collagen trimer, after reduction of disulfide bonds with DTT. (B) Two-dimensional gel electrophoresis of the proband's fibroblast cell layer collagens. Disulfide bonds were unreduced in the first dimension and reduced with DTT in the second dimension. The abnormal protein band in A, lane 2, was dissociated by DTT into proteins, designated $\alpha 1(\mathrm{I})^{\prime}$, which migrated in a similar manner to control $\alpha 1$ (I) chains.

these patients revealed variability in diameter and irregularity of dermal collagen fibrils, with granulofilamentous material visible along the collagen fibrils (similar to what is shown in Figure 4A), and collagen biosynthetic studies using fibroblast cultures bearing the R134C substitution also showed disulfide-bonded $\alpha 1$ (I) dimers within the fibroblasts. Thus, the ultrastructural and biosynthetic anomalies produced by the R134C and the R836C substitutions were similar. However, in contrast to the R134C substitution that was associated with a classical Ehlers-Danlos syndrome type I phenotype (soft, velvety, and hyperextensible skin; numerous atrophic paper scars of the skin; ecchymoses on the legs; and generalized joint hyperlaxity), the present R836C
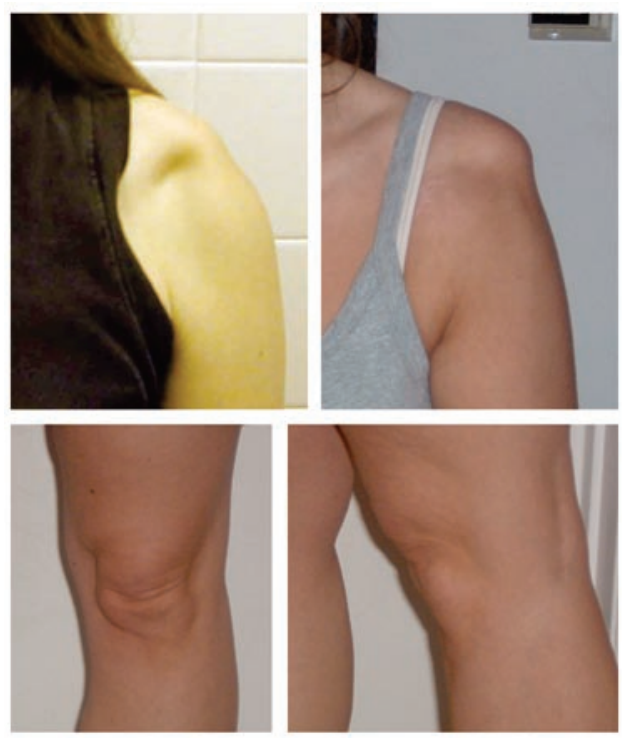
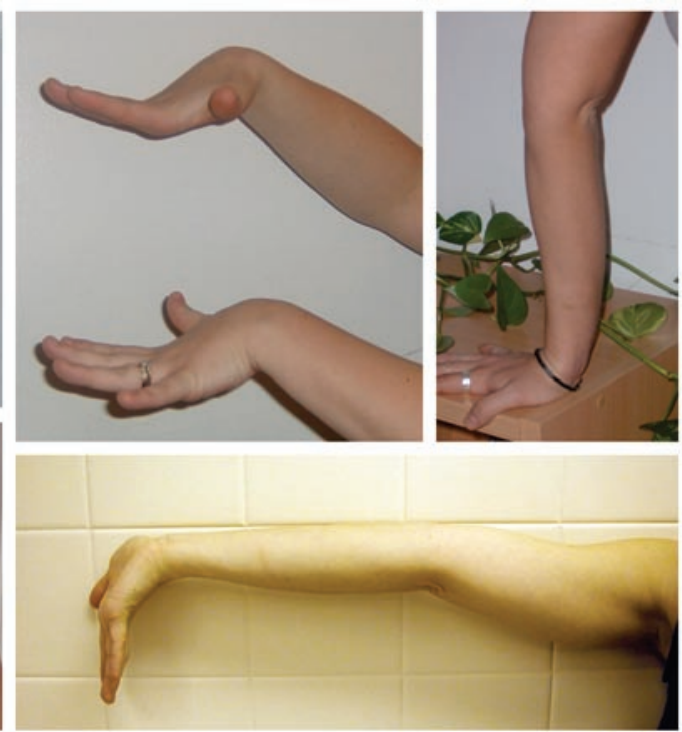

\section{Figure 6}

Voluntary subluxation of various joints in ADC patients. Photographs of 4 individuals carrying the R836C mutation (III-13, IV-2, IV-3, and IV-4 of family 1): upper left, 2 individuals with subluxation of the shoulders; lower left, 2 views of patellar subluxation; right: views of hyperextension and subluxation in the fingers and the elbow. 
substitution was associated with episodes of infantile cortical hyperostosis, and 2 of the 3 Caffey families showed features of Ehlers-Danlos syndrome type III (24). Interestingly, neither $\mathrm{R} \rightarrow \mathrm{C}$ mutation was associated with osteogenesis imperfecta.

There are several possible mechanisms by which the R836C mutation could result in the observed ADC phenotype. The analysis of collagen fibrils in skin fibroblasts showed increased disulfide crosslinking, either within or between mutant collagen fibrils, which may be responsible for the observed alterations in collagen architecture. Disulfide crosslinking may also occur between the mutant collagens and other cystein-containing proteins in the bone matrix. The Caffey mutation may furthermore disrupt an important site of interaction with other peptides or proteins. Arg836 is located within the carboxyterminal cyanogen bromide peptide 6 (CB6) of the $\alpha 1$ (I) chain, which has been shown to bind with high affinity to IL-2 and to the amyloid protein precursor (APP) (32-34). However, the CB6 peptide comprises 218 amino acid residues, i.e. approximately $21 \%$ of the $\alpha 1$ (I) chain (22), and the specific binding sites for IL-2 and APP within the CB6 peptide are currently unknown. It also is plausible that the $\mathrm{R} 836 \mathrm{C}$ substitution within the $\alpha 1$ (I) chain reduces the thermal stability of the collagen triple helix. Based on studies with a host-guest collagen peptide, Ac-(Gly-Pro-Hyp) ${ }_{3}$-Gly-X-Y-(GlyPro-Hyp)-Gly-Gly-CONH${ }_{2}$, in which an arginine residue had been replaced with a cysteine residue in the X position $(30,35)$, the $\mathrm{R} 836 \mathrm{C}$ mutation identified in Caffey patients would be predicted to decrease the melting temperature of the collagen triple helix by $4.5^{\circ} \mathrm{C}$. This change could provide another possible explanation for the observed alterations in collagen architecture. Last, prostaglandins may be involved in the development of hyperostosis, as short-term PGE1 or PGE2 administration for the treatment of ductal-dependent cardiac lesions typically leads to bone changes that resemble those present in ADC $(12-14,36)$. The inflammatory signs that often accompany the hyperostotic lesions in Caffey disease support this hypothesis. However, examination of additional ADC families, as well as in vitro and in vivo studies, are needed to help define the mechanism(s) that lead to the disease phenotype.

While the hyperostotic lesions in ADC occur only in infancy, the mutant $\alpha 1$ (I) chain is presumably present in the bone matrix throughout life. Based on our radiographic observations, detachment of the periosteum from the underlying bone appears to be a key pathological process in the onset of episodes of cortical hyperostosis. However, there was no consistent reporting of events in our families that may have precipitated this periosteal detachment. The only recurrent finding was the onset of episodes of cortical hyperostosis within the first 5 months of postnatal life, which suggests that infants in this age group may have an increased susceptibility to periosteal injury. Consistent with this hypothesis, a radiographic study of babies who died of sudden infant death syndrome between 1 and 4 months of age showed that mild subperiosteal new bone formation, which was distinct from the underlying diaphysis and metaphysis, had occurred in the long bones of $35 \%$ of the cases (37). Furthermore, large amounts of subperiosteal new bone characteristically accompany healing fractures (38), osteomyelitis (39), and scurvy $(40,41)$ in neonates and infants. In all of these conditions, the bone can enlarge in diameter by several fold but remodels over the following 2 years, similar to what occurs with the hyperostotic lesions in Caffey disease. In contrast, subperiosteal elevation is more lim- ited in older children with fractures and osteomyelitis (42). Thus, intrinsic differences in periosteal bone formation in infants and adults may explain the absence of hyperostotic lesions in adults with the R836C mutation. Furthermore, differences in the clinical threshold for periosteal detachment, influenced by the genetic background and/or environmental exposures, may explain the incomplete penetrance of the hyperostotic phenotype. Similar explanations have been provided to account for the widely differing fracture rates among affected individuals in families with classical osteogenesis imperfecta type I due to autosomal dominant COL1A1 haploinsufficiency $(26,43,44)$.

Our clinical evaluations of the affected individuals in the 3 families have extended the reported phenotype of Caffey disease (1). Individuals bearing the Caffey allele in 2 of the 3 families had features that were similar to those observed in patients with EhlersDanlos syndrome type III (24), and the absence of such features in the third family may be attributed to the current young age of the affected individuals. The relatively high number of fractures in family 1 suggested that bone fragility may also be part of the phenotype. However, bone densitometry studies in an affected adult patient (IV-2) were found to be normal, and similar fracture rates can occur in the normal population (45). Nonetheless, bone health of the ADC families requires further study, as radiographs taken during the episodes of cortical hyperostosis show that abnormalities in cortical and endosteal bone accompany the more obvious formation of subperiosteal new bone.

In summary, ADC is associated with a mutation in COL1A1 and thus belongs to the family of type I collagen-related conditions that include osteogenesis imperfecta types I-IV (46); Ehlers-Danlos syndrome types I $(31)$ and VII $(47,48)$ and a recessive cardiac valvular form (49); idiopathic osteoporosis (50); arterial dissections (51); and dermatofibrosarcoma protuberans, which was found to be associated with translocations between chromosomes 17 and 22 leading to the production of a fusion protein consisting of $\alpha 1$ (I) collagen and PDGF- $\beta$ (52). The findings in the present study extend the phenotypic spectrum of disorders associated with genetic changes in the type I collagen genes to include a hyperostotic disorder, but it remains uncertain how the R836C mutation results in a temporally and spatially limited defect in bone.

\section{Methods}

Families with ADC. A large, 3-generation Canadian ADC family (family 1) was ascertained, and historical details concerning episodes of infantile cortical hyperostosis were obtained from family members. Previous reports on this family also included detailed accounts of all episodes of possible infantile cortical hyperostosis $(7,8)$. These sources of information were combined in order to validate disease assignments within the pedigree. For the purposes of genetic linkage analysis, individuals in this family were designated as affected if they had radiographically proven Caffey disease (affected members) or if they had children diagnosed with Caffey disease (obligate carriers). Genomic DNA from 24 such individuals was available for study. Unrelated spouses were designated as unaffected.

Besides family 1, two additional small kindreds (families 2 and 3 ) were ascertained, which had 3 and 2 affected members, respectively. The small Canadian kindred (family 2) included the mother and her 2 children (see Figure 2C), who were clinically and radiographically affected by Caffey disease (see Figure 1), while the small Australian family (family 3) consisted of affected monozygotic twins, a healthy brother, and healthy parents (see Figure 2D) (19). A single sporadic case had hyperostosis of a rib, which was possibly due to ADC. Furthermore, 2 sporadic cases of the more severe 
prenatal form of Caffey disease were studied $(20,21)$. Autosomal recessive inheritance was postulated for the prenatal cases (21).

Detailed information was obtained from all families concerning possible events that may have precipitated episodes of infantile cortical hyperostosis. These enquiries were undertaken soon after the onset of the episodes of cortical hyperostosis in the affected children in families 2 and 3 as well as in some of the recently born affected members of the large family 1 . The long-term sequelae of the condition in clinically affected individuals and in obligate carriers were determined. The long-term clinical features of the individuals in each family that did not bear the Caffey allele were also recorded.

Genetic screening and DNA analysis. In family 1, we performed a genome-wide scan with microsatellite markers spaced at about 10-centimorgan intervals (Webber set 9; Invitrogen Corp.) followed by fine mapping using commercially available markers for the linked interval. We performed LOD score calculations using the Mlink program (http://linkage.rockefeller.edu/) assuming a disease frequency of $0.1 \%$, equal allele frequencies, and autosomal dominant inheritance with full penetrance. Candidate genes in the linked region were identified and sequenced by the Massachusetts General Hospital Sequencing Core using Applied Biosystems Taq DyeDeoxy Terminator cycle sequencing kits and methods.

Once the putative Caffey gene and its mutation were identified, we screened genomic DNA from affected individuals and obligate carriers of the various families, as well as the sporadic cases and controls, by restriction mapping of PCR products. For these studies, we used the forward primer AGCAGGGGAATATGGGTCAG and the reverse primer GGCCCTGAGAAAAACCATC to generate a 495-bp PCR product. The mutant PCR product lacked 1 of the cleavage sites for the restriction endonuclease HpyCH4IV, which thus allowed detection of the mutation by agarose gel electrophoresis after enzymatic digestion of the amplified DNA (wildtype, bands of 259, 60, and $176 \mathrm{bp}$; mutant, bands of 319 and $176 \mathrm{bp}$; note that the 60-bp band is not shown in Figure 2B). In individuals who did not bear the R836C mutation in COL1A1, mutations were sought in other parts of COL1A1 as well as in COL1A2, as described previously (53).

Informed consent was obtained from all study participants prior to their inclusion in these studies. These studies have been approved by the institutional review boards at the respective institutions and were performed in accordance with the guidelines of the Declaration of Helsinki.
Dermal bistology and fibroblast cultures. Skin biopsies were obtained from the proband (IV-2) of family 1 . We processed 1 biopsy for electron microscopy using previously described methods (54). The same methods were used to process the patient and control samples for electron microscopy. The second biopsy specimen was used to establish fibroblast cultures, which were grown to confluency in the presence of $\beta$-aminopropionitrile, which blocks the formation of lysine-derived cross-linkages, and ascorbate, which is required for normal prolyl- and lysyl-hydroxylation of collagen. The cultures were analyzed 5-7 days after plating, as previously described $(55,56)$. Fibroblast cultures from an age- and sex-matched individuals served as controls. Cell layer and medium fractions were analyzed separately, and the procollagens were converted to collagen by limited pepsin digestion. The collagens were resolved by 1-dimensional and 2-dimensional SDS-PAGE and stained with Coomassie G-250 (Invitrogen Corp.) (28). Where applicable, disulfide bonds were reduced with DTT.

\section{Acknowledgments}

We thank the affected individuals and their families for their assistance. We also thank C.S. Houston for his assistance with the radiology of Caffey disease. This work was supported by grants from the Canadian Institutes of Health Research and the Canadian Arthritis Network (to W.G. Cole); grants from the Foundation for Pediatric Research and the Paivikki and Sakari Sohlberg Foundation and a Research Fellowship from the European Society for Paediatric Endocrinology, sponsored by Novo Nordisk A/S (to O. Mäkitie); and NIH grants R01 46718-10 (to H. Jüppner) and K08 HD41512 (to R.C. Gensure).

Received for publication July 19, 2004, and accepted in revised form February 15, 2005.

Address correspondence to: Harald Jüppner, Endocrine and Pediatric Endocrine Units, Departments of Medicine and Pediatrics, Massachusetts General Hospital and Harvard Medical School, Boston, Massachusetts, USA. Phone: (617) 726-3966; Fax: (617) 726-7543; E-mail: jueppner@helix.mgh.harvard.edu.
1. Caffey, J. 1957. Infantile cortical hyperostosis; a review of the clinical and radiographic features. Proc. R. Soc. Med. 50:347-354.

2. Bernstein, R.M., and Zaleske, D.J. 1995. Familial aspects of Caffey's disease. Am. J. Orthop. 24:777-781.

3. Caffey, J. 1952. On some late skeletal changes in chronic infantile cortical hyperostosis. Radiology. 59:651-657.

4. Blank, E. 1975. Recurrent Caffey's cortical hyperostosis and persistent deformity. Pediatrics. 55:856-860.

5. Heyman, E., Laver, J., and Beer, S. 1982. Prostaglandin synthetase inhibitor in Caffey disease. J. Pediatr 101:314.

6. Bush, L., and Merrell, O. 1952. Infantile cortical hyperostosis. Report of a case responding to treatment with corticotropin. J. Pediatr. 40:330-333.

7. Gerrard, J.W., Holman, G.H., Gorman, A.A., and Morrow, I.H. 1961. Familial infantile cortical hyperostosis. J. Pediatr. 59:543-548.

8. Maclachlan, A.K., Gerrard, J.W., Houston, C.S., and Ives, E.J. 1984. Familial infantile cortical hyperostosis in a large Canadian family. Can. Med. Assoc. J. 130:1172-1174.

9. Van Buskirk, F.W., Tampas, J.P., and Peterson, O.S., Jr. 1961. Infantile cortical hyperostosis; an inquiry into its familial aspects. Am. J. Roentgenol. Radium Ther. Nucl. Med. 85:613-632.

10. Barba, W.P., and Freriks, D.J. 1953. The familial occurrence of infantile cortical hyperostosis in utero. J. Pediatr. 42:141-150.

11. Saul, R.A., Lee, W.H., and Stevenson, R.E. 1982. Caffey's disease revisited. Further evidence for autosomal dominant inheritance with incomplete penetrance. Am. J. Dis. Child. 136:55-60.

12. Parker, S., and Griffiths, H. 1990. Prostaglandin-induced cortical hyperostosis. Orthopedics. 13:243-244

13. Kaufman, M., and El-Chaar, G. 1996. Bone and tissue changes following prostaglandin therapy in neonates. Ann. Pharmacother. 30:269-274, 277.

14. Woo, K., Emery, J., and Peabody, J. 1994. Cortical hyperostosis: a complication of prolonged prostaglandin infusion in infants awaiting cardiac transplantation. Pediatrics. 93:417-420.

15. Snedecor, S., Knapp, R., and Wilson, H. 1935. Traumatic ossifying periostitis of the newborn. Surg. Gynecol. Obstet. 61:385-387.

16. Rothman, P., and Leon, E. 1948. Hypervitaminosis A. Report of two cases in infants. Radiology. 51:368-374.

17. Talab, Y., and Mallouh, A. 1988. Hyperostosis with hyperphosphatemia: a case report and review of the literature. J. Pediatr. 114:1010-1013.

18. Caffey, J. 1946. Infantile cortical hyperostosis. J. Pediatr. 29:541-559.

19. Couper, R.T., McPhee, A., and Morris, L. 2001. Indomethacin treatment of infantile cortical periostosis in twins. J. Paediatr. Child Health. 37:305-308.
20. Dahlstrom, J.E., et al. 2001. Lethal prenatal onset infantile cortical hyperostosis (Caffey disease). Pathology. 33:521-525.

21. Schweiger, S., et al. 2003. Antenatal onset of cortical hyperostosis (Caffey disease): case report and review. Am. J. Med. Genet. 120A:547-552.

22. Dalgleish, R. 1997. The human type I collagen mutation database. Nucleic Acids Res. 25:181-187.

23. Drvaric, D.M., et al. 1989. Prostaglandin-induced hyperostosis. A case report. Clin. Orthop. 246:300-304.

24. Beighton, P., De Paepe, A., Steinmann, B., Tsipouras, P., and Wenstrup, R.J. 1998. Ehlers-Danlos syndromes: revised nosology, Villefranche, 1997. Ehlers-Danlos National Foundation (USA) and Ehlers-Danlos Support Group (UK). Am. J. Med. Genet. 77:31-37.

25. Byers, P.H., and Steiner, R.D. 1992. Osteogenesis imperfecta. Annu. Rev. Med. 43:269-282.

26. Cole, W.G. 2002. Advances in osteogenesis imperfecta. Clin. Orthop. 401:6-16.

27. Chan, D., Rogers, J.F., Bateman, J.F., and Cole, W.G. 1995. Recurrent substitutions of arginine 789 by cysteine in pro-alpha 1 (II) collagen chains produce spondyloepiphyseal dysplasia congenita. J. Rheumatol. Suppl. 43:37-38.

28. Chan, D., Taylor, T.K., and Cole, W.G. 1993. Characterization of an arginine 789 to cysteine substitution in alpha 1 (II) collagen chains of a patient with spondyloepiphyseal dysplasia. J. Biol. Chem. 
268:15238-15245.

29. Tomso, D.J., and Bell, D.A. 2003. Sequence context at human single nucleotide polymorphisms: overrepresentation of $\mathrm{CpG}$ dinucleotide at polymorphic sites and suppression of variation in $\mathrm{CPG}$ islands. J. Mol. Biol. 327:303-308.

30. Persikov, A.V., Ramshaw, J.A., Kirkpatrick, A., and Brodsky, B. 2000. Amino acid propensities for the collagen triple-helix. Biochemistry. 39:14960-14967.

31. Nuytinck, L., et al. 2000. Classical Ehlers-Danlos syndrome caused by a mutation in type I collagen. Am. J. Hum. Genet. 66:1398-1402.

32. Beher, D., Hesse, L., Masters, C.L., and Multhaup, G. 1996. Regulation of amyloid protein precursor (APP) binding to collagen and mapping of the binding sites on APP and collagen type I. J. Biol. Chem. 271:1613-1620.

33. Somasundaram, R., et al. 2000. Collagens serve as an extracellular store of bioactive interleukin 2. J. Biol. Chem. 275:38170-38175.

34. Di Lullo, G.A., Sweeney, S.M., Korkko, J., Ala-Kokko, L., and San Antonio, J.D. 2002. Mapping the ligandbinding sites and disease-associated mutations on the most abundant protein in the human, type I collagen. J. Biol. Chem. 277:4223-4231.

35. Persikov, A.V., Ramshaw, J.A., and Brodsky, B. 2000. Collagen model peptides: sequence dependence of triple-helix stability. Biopolymers. 55:436-450.

36. Velaphi, S., et al. 2004. Cortical hyperostosis in an infant on prolonged prostaglandin infusion: case report and literature review. J. Perinatol. 24:263-265.

37. Kwon, D.S., Spevak, M.R., Fletcher, K., and Kleinman, P.K. 2002. Physiologic subperiosteal new bone formation: prevalence, distribution, and thickness in neonates and infants. AJR Am. J. Roentgenol. 179:985-988.

38. Morris, S., Cassidy, N., Stephens, M., McCormack, D., and McManus, F. 2002. Birth-associated femoral fractures: incidence and outcome. J. Pediatr. Orthop. 22:27-30.

39. Abernethy, L.J., Lee, Y.C., and Cole, W.G. 1993.
Ultrasound localization of subperiosteal abscesses in children with late-acute osteomyelitis. J. Pediatr. Orthop. 13:766-768.

40. Front, D., Hardoff, R., Levy, J., and Benderly, A. 1978. Bone scintigraphy in scurvy. J. Nucl. Med. 19:916-917. 41. Ratanachu-Ek, S., Sukswai, P., Jeerathanyasakun, Y., and Wongtapradit, L. 2003. Scurvy in pediatric patients: a review of 28 cases. J. Med. Assoc. Thai. 86(Suppl. 3):S734-S740.

42. Cole, W.G., Dalziel, R.E., and Leitl, S. 1982. Treatment of acute osteomyelitis in childhood. J. Bone Joint Surg. Br. 64:218-223.

43. Willing, M.C., Deschenes, S.P., Slayton, R.L., and Roberts, E.J. 1996. Premature chain termination is a unifying mechanism for COL1A1 null alleles in osteogenesis imperfecta type I cell strains. Am. J. Hum. Genet. 59:799-809.

44. Willing, M.C., et al. 1994. Osteogenesis imperfecta type I: molecular heterogeneity for COL1A1 null alleles of type I collagen. Am. J. Hum. Genet. 55:638-647.

45. Jones, I.E., Williams, S.M., Dow, N., and Goulding, A. 2002. How many children remain fracture-free during growth? a longitudinal study of children and adolescents participating in the Dunedin Multidisciplinary Health and Development Study. Osteoporos. Int. 13:990-995.

46. Byers, P.H., and Cole, W.G. 2002. Osteogenesis imperfecta. In Connective tissue and its heritable disor ders molecular, genetic and medical aspects. P.M. Royce and B. Steinmann, editors. Wiley-Liss. New York, New York, USA. 385-430.

47. Cole, W.G., Chan, D., Chambers, G.W., Walker, I.D., and Bateman, J.F. 1986. Deletion of 24 amino acids from the pro-alpha 1 (I) chain of type I procollagen in a patient with the Ehlers-Danlos syndrome type VII. J. Biol. Chem. 261:5496-5503.

48. Byers, P.H., et al. 1997. Ehlers-Danlos syndrome type VIIA and VIIB result from splice-junction mutations or genomic deletions that involve exon 6 in the COL1A1 and COL1A2 genes of type I col- lagen. Am. J. Med. Genet. 72:94-105.

49. Schwarze, U., et al. 2004. Rare autosomal recessive cardiac valvular form of Ehlers-Danlos syndrome results from mutations in the COL1A2 gene that activate the nonsense-mediated RNA decay pathway. Am.J. Hum. Genet. 74:917-930.

50. Grant, S.F., et al. 1996. Reduced bone density and osteoporosis associated with a polymorphic Sp1 binding site in the collagen type I alpha 1 gene. Nat. Genet. 14:203-205.

51. Mayer, S.A., Rubin, B.S., Starman, B.J., and Byers, P.H. 1996. Spontaneous multivessel cervical artery dissection in a patient with a substitution of alanine for glycine (G13A) in the alpha 1 (I) chain of type I collagen. Neurology. 47:552-556.

52. Simon, M.P., et al. 1997. Deregulation of the platelet-derived growth factor B-chain gene via fusion with collagen gene COL1A1 in dermatofibrosarcoma protuberans and giant-cell fibroblastoma. Nat. Genet. 15:95-98.

53. Korkko, J., Annunen, S., Pihlajamaa, T., Prockop, D.J., and Ala-Kokko, L. 1998. Conformation sensitive gel electrophoresis for simple and accurate detection of mutations: comparison with denaturing gradient gel electrophoresis and nucleotide sequencing. Proc. Natl. Acad. Sci. U. S. A. 95:1681-1685.

54. Cole, W.G., Evans, R., and Sillence, D.O. 1987. The clinical features of Ehlers-Danlos syndrome type VII due to a deletion of 24 amino acids from the pro alpha $1(\mathrm{I})$ chain of type I procollagen. J. Med. Genet. 24:698-701.

55. Chan, D., Lamande, S.R., Cole, W.G., and Bateman, J.F. 1990. Regulation of procollagen synthesis and processing during ascorbate-induced extracellular matrix accumulation in vitro. Biochem. J. 269:175-181.

56. Hata, R., and Senoo, H. 1989. L-ascorbic acid 2 phosphate stimulates collagen accumulation, cell proliferation, and formation of a three-dimensional tissuelike substance by skin fibroblasts. J. Cell. Physiol. 138:8-16. 